\title{
Nachweis der 27 Geraden auf der allgemeinen Oberfläche dritter Ordnung.
}

\author{
(Von Herrn H. Schröter zu Breslau.)
}

Unter den verschiedenen Erzeugungsarten der Flächen dritten Grades, welche Steiner ${ }^{*}$ ) angegeben und aus denen er eine Reihe der wesentlichsten Eigenschaften dieser Flächen hergeleitet hat, ist eine nicht angeführt, welche als ursprünglicher gelten kann und für manche Untersuchungen vortheilhafter ist; diese, wie es scheint, zuerst von Grassmann ${ }^{* *}$ ) ausgesprochene Erzeugungsart lautet dort:

Der Durchschnitt dreier einander projektivischen Ebenenbiischel zweiter Stufe ist eine Oberfläche dritter Ordnung.

Der Nachweis, dass die auf diese Art durch Punkte in linearer Weise construirte Fläche jene 27 Geraden enthält, welche auf der allgemeinen Oberfläche dritter Ordnung liegen, bietet sich bei dieser Erzeugungsart nicht so unmittelbar dar, wie bei den anderen, weil in letzteren meist schon eine Gerade als der Oberfläche angehörig in die Definition eingeht; zugleich führt die Auffindung der 27 Geraden hier direkt auf jenes anschauliche Arrangement dieser Linien, auf welches Schläffli ${ }^{* *}$ ) zuerst aufmerksam gemacht hat und endlich sind die Erzeugungsarten von Steiner wie auch die von $\dot{F}$. August ${ }^{* * * *}$ ) angegebene in der obigen enthalten. Die Auffindung der 27 Geraden auf der Oberfläche dritter Ordnung, als Erzeugniss dreier collinearen Ebenenbündel aufgefasst, ist der Gegenstand dieses Aufsatzes und dürfte aus den angegebenen Gründen nicht ohne Interesse sein, obschon die Frage von anderen Gesichtspunkten aus sowohl durch rein geometrische Betrachtungen als auch durch die analytischen Untersuchungen von Cayley, Salmon und Clebsch als erledigt angesehen werden darf. Aus der obigen Definition ergiebt sich auch eine

*) Dieses Journal Bd. 53, Seite 133.

**) Dieses Journal Bd. 49, Seite 64 .

***) The quarterly Journal vol. II, pag. 116.

****) Disquisitiones de superficiebus tertii ordinis. Inaug. Diss. 1862.

Journal für Mathematik Bd.LXII. Heft 3. 
lineare Construction der Oberfläche dritter Ordnung durch 19 im Raume beliebig gegebene Punkte, auf welche ich bei einer anderen Gelegenheit zurückzukommen gedenke.

1. Alle durch einen Punkt gehenden Ebenen sollen ein Ebenenbündel heissen zur Unterscheidung von Ebenenbiischel, welches nur die durch eine Gerade gehenden Ebenen enthält. Die Ebenen zweier Ebenenbündel können eindeutig auf einander bezogen werden, so dass jeder Ebene des einen Bündels eine einzige bestimmte des anderen entspricht und umgekehrt. Diese bekannte Beziehung (Collinearität) ist die Ausdehnung der projectivischen Beziehung auf Gebilde von doppelt unendlicher Mächtigkeit und kann nach Seidewitz ${ }^{*}$ ) folgendermaassen hergestellt werden: Sei $M$ der Mittelpunkt des einen, $M^{\prime}$ der des anderen Bündels, seien durch $M$ zwei beliebige Ebenen $a, b$ angenommen und durch $M^{\prime}$ zwei beliebige Ebenen $a^{\prime}, b^{\prime}$, ferner in der Ebene $a$ ein gewöhnliches Strahlbüschel mit dem Mittelpunkt $M$, in der Ebene $a^{\prime}$ aber ein mit diesem projectivisches Strahlbüschel um den Mittelpunkt $M^{\prime}$, in gleicher Weise in $b$ ein beliebiges Strahlbüschel um $M$ und in $b^{\prime}$ ein projectivisches Strahlbüschel um $M^{\prime}$; endlich sei noch bei dieser zwiefachen sonst willkürlich festgestellten projectivischen Beziehung die eine Bedingung erfüllt, dass der als Schnittlinie der Ebenen $a, b$ in beiden Strahlbüscheln auftretende Strahl zu seinem entsprechenden für beide Beziehungen denselben als Schnittlinie von $a^{\prime} b^{\prime}$ auftretenden Strahl habe; alsdann ist die collineare Beziehung der beiden Ebenenbündel $M$ und $M^{\prime}$ vollständig bestimmt, denn irgend eine durch $M$ gelegte Ebene $x$ schneidet die Ebenen $a$ und $b$ längs zwei Strahlen, deren entsprechende in den Ebenen $a^{\prime} b^{\prime}$ die entsprechende Ebene $x^{\prime}$ bestimmen.

Es ergiebt sich hieraus unmittelbar, dass irgend vier als entsprechend willkürlich festgesetzte Paare von Ebenen (von denen sich nicht je drei in derselben Geraden schneiden) zur Bestimmung der collinearen Beziehung beider Bündel ausreichen und erforderlich sind, dass aus ihnen zu jeder fünften Ebene des einen Bündels die entsprechende des anderen auf lineare Weise zu construiren ist, dass, wenn die Ebene $x$ ein gewöhnliches Ebenenbüschel beschreibt, die entsprechende $x^{\prime}$ ein projectivisches Ebenenbüschel beschreibt

*) F. Seidewitz, Darstellung der geometrischen Verwandtschaften mittelst projectivischer Gebilde, Grunerts Archiv für Math. u. Phys. Bd. VII, Seite 113 und Bd.VIII, Seite $1 \mathrm{ff}$. 
u. s. f., wie dies denn aus der collinearen Beziehung überhaupt hinlänglich bekannt ist *). Gleichzeitig stecken in den beiden collinearen Ebenenbündeln zwei collineare Strahlenbündel (Raumbüschel), denn jedem durch $M$ gehenden Strahle, als Schnittlinie zweier beliebigen Ebenen aufgefasst, entspricht ein bestimmter durch $M^{\prime}$ gehender Strahl des anderen Bündels, die Schnittlinie der beiden jenen entsprechenden Ebenen, und es treten also bei dieser collinearen Beziehung die beiden Gebilde eines Strahlenbündels und Ebenenbündels gleichzeitig auf. Fügt man ihnen noch die nach dem Prinzip der Polarität zugehörigen beiden Gebilde doppelter Unendlichkeit hinzu: Die Ebene erfüllt einerseits mit Punkten, andererseits mit Geraden, und überträgt auch auf sie das oben angedeutete Prinzip der eindeutigen Beziehung der Elemente verschiedener Gebilde auf einander, so hat man die Grundelemente für die Untersuchung räumlicher Gestalten von grosser Mannigfaltigkeit und eine reiche Quelle zur Auffindung geometrischer Wahrheiten, welche zuerst aufgedeckt zu haben Steiners Verdienst ist. Wir beschränken uns hier auf die Oberfläche dritter Ordnung.

2. Drei beliebig im Raume liegende collineare Ebenenbündel erzeugen eine allgemeine Oberfläche dritter Ordnung, d. h. der Ort des Schnittpunktes dreier entsprechenden Ebenen der drei Bündel ist diese Oberfläche dritter Ordnung; sie geht, wie ersichtlich ist, selbst durch die Mittelpunkte der Bündel hindurch. Um den Grad der Oberfläche zu ermitteln, wollen wir die Durchschnittscurve des eben definirten Ortes mit einer beliebigen Transversalebene aufsuchen und zeigen, dass dies eine allgemeine Curve dritten Grades ist. Enthält nämlich die Transversalebene $E$ einen Punkt des Ortes, so müssen drei entsprechende Ebenen der drei collinearen Bündel die Ebene $E$ längs dreier Geraden schneiden, welchẹ in diesen Punkt zusammenlaufen. Ziehen wir nun durch den Mittelpunkt $M_{1}$ des ersten Bündels einen beliebigen Strahl $s_{1}$, so entspricht demselben ein bestimmter Strahl $s_{2}$ des zweiten Bündels $M_{2}$ und ein bestimmter Strahl $s_{3}$ des dritten Bündels $M_{3}$; mögen $s_{1} s_{2} s_{3}$ der Ebene $E$ in $p_{1} p_{2} p_{3}$ begegnen. Drehen wir um $s_{1}$ eine Ebene, so drehen sich die entsprechenden Ebenen in den beiden anderen Bündeln um $s_{2}$ und $s_{3}$ und beschreiben mit dem ersteren projectivische Ebenenbüschel, ihre Durchschnitte also projectivische Strahlbüschel; die beiden Strahlbüschel um $p_{1}$ und $p_{2}$ haben

*) Vergl. Möbius barycentrischer Kalkul Seite $301 \mathrm{ff.}$ und Magnus Sammlung von Aufgaben und Lehrsätzen aus der analytischen Geometrie $\mathrm{II}^{\text {ter }}$ Theil Seite $31 \mathrm{ff}$. 
als Ort der Durchschnittspunkte entsprechender Strahlen einen Kegelschnitt $\boldsymbol{K}$ und die Strahlbüschel um $p_{1}$ und $p_{3}$ erzeugen in gleicher Weise einen Kegelschnitt $K^{\prime}$; diese beiden Kegelschnitte $K$ und $K^{\prime}$, welche den Punkt $p_{1}$ gemein haben, schneiden sich ausserdem im Allgemeinen noch in drei Punkten, die der Construction zufolge die Eigenschaft besitzen müssen, dass durch jeden derselben drei entsprechende Ebenen der drei collinearen Bündel gehen; es sind also Punkte des gesuchten Ortes, die in der Ebene $E$ liegen. Von jedem der beiden Kegelschnitte $\boldsymbol{K}$ und $\boldsymbol{K}^{\prime}$ lassen sich ausserdem drei Punkte angeben, die von Wichtigkeit sind; die beiden collinearen Bündel $M_{1}$ und $M_{2}$ als Strahlenbündel aufgefasst treffen nämlich die doppelt gedachte Transversalebene $E$ in zwei auf einander liegenden collinearen Punktgebilden, die im Allgemeinen drei Doppelpunkte $P Q R$ besitzen d. h. drei solche Punkte, in deren jedem zwei entsprechende Punkte der beiden collinearen Ebenen vereinigt sind $\left.{ }^{*}\right)$. Es ist klar, dass der Kegelschnitt $K$ durch die Punkte $P$, $Q, R$ hindurchgehen muss, weil $\left(M_{1} p_{1} P\right)$ und $\left(M_{2} p_{2} P\right)$ entsprechende Ebenen sind u. s. f. Ebenso treffen die collinearen Bündel $M_{1}$ und $M_{3}$ die doppelt gedachte Ebene $E$ in zwei collinearen auf einander liegenden ebenen Punktgebilden, welche drei Doppelpunkte $P^{\prime} Q^{\prime} R^{\prime}$ besitzen, und der Kegelschnitt $K^{\prime}$ muss durch dieselben hindurchgehen. Denken wir uns jetzt den Strahl $s_{1}$ in einer beliehigen aber festen durch $M_{1}$ gehenden Ebene $E_{1}$ um den Mittelpunkt $M_{1}$ gedreht, dann wird der Strahl $s_{2}$ sich in der entsprechenden durch $M_{2}$ gehenden Ebene $E_{2}$ um $M_{2}$ drehen, und ebenso $s_{3}$ in der entsprechenden Ebene $E_{3}$ um $M_{3}$ herumbewegen; und alle drei werden projectivische Strahlbüschel beschreiben; werde die Transversalebene $E$ von den drei entsprechenden Ebenen $E_{1} E_{2} E_{3}$ längs dreier Geraden $l_{1} l_{2} l_{3}$ geschnitten, dann geht offenbar der Kegelschnitt $K$ durch den Schnittpunkt $S$ von $l_{1}$ und $l_{2}$ und ebenso $K^{\prime}$ durch den Schnittpunkt $S^{\prime}$ von $l_{1}$ und $l_{3}$. Mit dem Strahle $s_{1}$ verändern sich nun auch gleichzeitig die beiden Kegelschnitte $K$ und $K^{\prime}$, aber der erstere läuft beständig durch die vier unveränderlichen Punkte $P Q R S$ und der letztere durch $P^{\prime} Q^{\prime} R^{\prime} S^{\prime}$; wir erhalten also zwei Kegelschnittbüschel von je vier Punkten und es müssen dieselben projectivisch sein, weil die Verbindungslinie $S S^{\prime}$ (oder $l_{l}$ ) von je zwei zusammengehörigen in derselben Punktreihe $p_{1}$ getroffen wird. Folglich ist der Ort der Schnittpunkte je zweier entsprechenden Kegelschnitte dieser beiden projectivischen Kegelschnittbüschel eine

*) Siehe Seidewitz a. a. O. 
allgemeine Curve dritten Grades, welche durch die sechs Punkte $P Q \boldsymbol{R} \boldsymbol{P}^{\prime} \boldsymbol{Q}^{\prime} \boldsymbol{R}^{\prime}$ (aber nicht durch $S$ und $S^{\prime}$ ) hindurchgeht ${ }^{*}$ ). Diese allgemeine Curve dritten Grades ist also der Ort solcher Punkte der Ebene $E$, in welchen sich drei entsprechende Ebenen der drei collinearen Bündel treffen d. h. die Schnittcurve der Ebene $E$ mit dem von den drei Bündeln erzeugten räumlichen Orte; dieser selbst ist also eine Oberfläche dritter Ordnung, w. z. b. w. Es ist zugleich für das Folgende von Wichtigkeit, zu wissen, welchen Ort diejenigen besonderen Ebenen je eines Bündels umhüllen, deren entsprechende in den beiden anderen sie längs den Punkten der eben gefundenen Curve dritten Grades in der Transversalebene $E$ schneiden. Um dies zu ermitteln haben wir nur die drei übrigen Schnittpunkte der beiden Kegelschnitte $\boldsymbol{K}$ und $\boldsymbol{K}^{\prime}$, welche $x y z$ heissen mögen, mit $p_{1}$ zu verbinden und aufzusuchen, von der wievielsten Classe der gesammte Ort der drei Verbindungslinien $p_{1} x, p_{1} y, p_{1} z$ wird. Denken wir uns hierzu einen beliebigen Punkt $\Pi$ der Ebene $E$ mit dem veränderlichen Punkte $p_{1}$ verbunden und suchen den zweiten Schnittpunkt dieses Strahls und des Kegelschnitts $K$ auf, so ist der Ort desselben, wie leicht zu sehen ist, ein neuer Kegelschnitt $\mathcal{R}$, welcher durch $\Pi$ geht; ebenso erhalten wir als Ort des Schnittpunktes von $\Pi p_{1}$ mit dem Kegelschnitt $K^{\prime}$ einen neuen Kegelschnitt $\Omega^{\prime}$, welcher gleichfalls durch $\Pi$ geht. Die beiden Kegelschnitte $\mathfrak{\Omega}$ und $\Re^{\prime}$ haben im Allgemeinen noch drei gemeinsame Punkte, die mit $\Pi$ verbunden drei Strahlen von der gewünschten Eigenschaft liefern; folglich ist der gesuchte Ort von der dritten Classe. Wir schliessen also Folgendes: Von den Schnittpunkten entsprechender Ebenen dreier collinearen Ebenenbündel liegen in einer beliebig gegebenen Transversalebene unendlich viele auf einer allgemeinen Curve dritten Grades und diejenigen je drei Ebenen, welche diese Punkte bestimmen, umhïllen in jedem der drei Bündel einen Kegel dritter Classe.

3. Drei entsprechende Ebenen der drei collinearen Bündel schneiden sich immer in einem Punkte, der ihrem Erzeugnisse, der Oberfläche dritter Ordnung, angehört; es kann aber insbesondere vorkommen, dass sie sich in derselben Geraden schneiden, in welchem Falle diese ganz der Oberfläche angehören muss. Um zu untersuchen, wie oft dies vorkommt, denken wir uns zwei verchiedene Transversalebenen $E$ und $E^{\prime}$ beliebig angenommen, dann 16 août 1853 . 
müsste ein solches besonderes Tripel von entsprechenden Ebenen einen Schnittpunkt sowohl in $E$ als auch in $E^{\prime}$ haben und umgekehrt. Nun umhüllen nach 2. diejenigen Ebenen des ersten Bündels, welche Punkte der Ebene $E$ liefern, einen Kegel dritter Classe und ebenso diejenigen Ebenen des ersten Bündels, welche Punkte der Ebene $E^{\prime}$ liefern. Diese beiden Kegel mit demselben Mittelpunkte $M_{1}$ haben im Allgemeinen neun gemeinschaftliche Berührungsebenen, von denen drei sofort anzugeben sind. In die Schnittlinie von $E$ und $E^{\prime}$ fällt nämlich dreimal ein Schnittpunkt dreier entsprechenden Ebenen hinein und dieser liegt dann zwar in beiden Transversalebenen, ist aber derselbe. Die übrigen sechs gemeinschaftlichen Berührungsebenen müssen dagegen solche Ebenen des ersten Bündels sein, dass jede mit ihren beiden entsprechenden im zweiten und dritten Bündel durch dieselbe Gerade geht. Wir schliessen also: Bei drei beliebig gegebenen collinearen Ebenenbiindeln kommt es im Allgemeinen sechs Mal vor, dass drei entsprechende Ebenen sich in derselben Geraden schneiden.

4. Die sechs geraden Linien, welche wir als vollständig der Oberfläche dritter Ordnung angehörig gefunden haben, geben zu neuen Geraden Veranlassung, die ebenfalls gänzlich auf der Oberfläche liegen. Jene ursprünglichen sechs Geraden sind aber selbst gewissen Bedingungen unterworfen, die sich unmittelbar herausstellen. Es ist einłeuchtend, dass sich im Allgemeinen keine zwei von ihnen treffen werden, denn sonst müssten sich drei entsprechende Strahlen der drei collinearen Bündel in einem Punkte treffen; dies würde aber eine Bedingung für die drei ganz willkürlich gegebenen collinearen Bündel sein, die nicht angenommen ist. Es können auch keine vier von jenen sechs Geraden die Generatrices derselben Art eines Hyperboloids sein, d. h. von drei Geraden gleichzeitig getroffen werden, denn sonst würde die ganze Schaar von Geraden, welche jene vier schnitten, der Oberfläche dritter Ordnung angehören müssen, diese würden also zerfallen in ein Hyperboloid und eine Ebene. Dagegen werden vier beliebige Gerade im Raume, von denen keine zwei sich begegnen und die auch nicht auf einem Hyperboloid liegen, bekanntlich immer im Allgemeinen von zwei bestimmten Geraden gleichzeitig getroffen, die auf bekannte Weise zu construiren sind; solche Gerade müssen der Oberfläche dritter Ordnung ganz angehören, weil sié vier Punkte mit ihr gemein haben.

Mögen die sechs ursprünglichen Geraden mit

$$
\begin{array}{llllll}
a & b & c & d & e & f
\end{array}
$$


und die Fläche dritten Grades zur Abkürzung mit $F^{(3)}$ bezeichnet werden; nehmen wir dann irgend vier $a b c d$ von ihnen und construiren die beiden Geraden $\alpha$ und $\beta$, welche ihnen gleichzeitig begegnen, so wird das durch die drei Geraden $a b$ bestimmte Hyperboloid $H^{(2)}$ auch $\alpha$ und $\beta$ enthalten und also mit $F^{(3)}$ fünf gerade Linien gemein haben, folglich nothwendig noch eine sechste Gerade, die wir mit $\gamma$ bezeichnen wollen, und welche nach dem Obigen nicht mit $a b c$ zu derselben Schaar Generatrices gehören kann, sondern zu der anderen Schaar gehören, also $a b c$ gleichzeitig schneiden muss. Mehr als diese sechs Geraden $a b c c \alpha \beta \gamma$ kann aber das Hyperboloid $\boldsymbol{H}^{(2)}$ mit $F^{(3)}$ nicht gemein haben. Denken wir uns daher die beiden Geraden $\alpha^{\prime} \beta^{\prime}$ construirt, welche $a b c$ e gleichzeitig treffen und endlich noch die beiden Geraden $\alpha^{\prime \prime} \beta^{\prime \prime}$, welche $a b c$ gleichzeitig treffen, so müssen diese vier Geraden $\alpha^{\prime} \beta^{\prime} \alpha^{\prime \prime} \beta^{\prime \prime}$, weil sie nothwendig sowohl dem Hyperboloid $H^{(2)}$ wie der Fläche $F^{(3)}$ angehören, in irgend einer Weise mit den dreien $\alpha \beta \gamma$ coincidiren. Von den beiden Geraden $\alpha^{\prime} \beta^{\prime}$ muss hiernach mindestens eine mit $\alpha$ oder $\beta$ coincidiren, denn fielen sie selbst zusammen und coincidirten mit $\gamma$, so hätten $\boldsymbol{H}^{(2)}$ und $\boldsymbol{F}^{(3)}$ diese doppelte Gerade und ausserdem noch fünf Gerade gemein, was unmöglich ist. Wir können also annehmen, dass $\alpha^{\prime}$ mit $\alpha$ zusammenfällt, d. h. mindestens eine der beiden Geraden, welche $a b c d$ treffen, muss auch $e$ treffen und da wir vier ganz beliebige aus den anfänglichen sechs Geraden herausgenommen haben, so gilt dies für jede vier; es stellt sich also folgende Bedingung, welcher die sechs anfänglichen Geraden unterworfen sind, heraus: Die sechs Geraden $a b$ c $d$ e $f$ liegen derart, dass eine von solchen zwei Geraden, welche irgend vier von jenen treffen, zugleich einer der beiden übrigen begegnet. Wir können aber weiter einsehen, dass nicht gleichzeitig $\alpha^{\prime}$ mit $\alpha$ und $\beta^{\prime}$ mit $\beta$ coincidiren kann, dass also $\beta^{\prime}$ mit $\gamma$ zusammenfallen muss; denn fielen $\alpha^{\prime}$ und $\alpha, \beta^{\prime}$ und $\beta$ zusammen, so müsste von den beiden letzten Geraden $\alpha^{\prime \prime} \beta^{\prime \prime}$ eine, etwa $\alpha^{\prime \prime}$, mit $\gamma$ und die andere $\beta^{\prime \prime}$ mit $\alpha$ oder $\beta$ zusammenfallen, d. h. die Geraden $\alpha \beta \gamma$ träfen in folgender Weise die anfänglichen sechs Geraden

$$
\begin{array}{lllllll}
\alpha & \ldots & a & b & c & d & e \\
\beta & \ldots & a & b & c & d & e f \\
\gamma & \ldots & a & b & c & f .
\end{array}
$$

Dies ist aber unmöglich, denn das durch die drei Geraden $a b$ bestimmte Hyperboloid $H^{(2)}$ begegnet nothwendig ausserdem der Fläche $F^{(3)}$ in drei unveränderlichen Geraden $\alpha \beta \gamma$; dieselben drei Geraden müssten also auch 
resultiren, wenn $f$ mit $d$ vertauscht würde oder überhaupt irgend eine Vertauschung der Geraden $d$ e f einträte, d. h. $\alpha \beta \gamma$ müssten auch in irgend einer Reihenfolge derart den anfänglichen sechs Geraden begegnen

$\begin{array}{llllll}\text { eine } & a & b & c & e & f \\ \text { die andere } & a & b & c & d & e\end{array}$

Bei der Identificirung dieses Arrangements mit dem vorigen würde aber immer folgen, dass vier von den Geraden $a b c$ d e f durch dieselben drei $\alpha \beta \gamma$ getroffen werden, was gegen die Annahme ist. Es bleibt also nur noch übrig, dass $\alpha^{\prime}$ mit $\alpha$ coincidirt, $\beta^{\prime}$ dagegen mit $\gamma$ und ebenso von den beiden letzten $\alpha^{\prime \prime}$ und $\beta^{\prime \prime}$ eine, etwa $\beta^{\prime \prime}$, mit $\gamma$ und die andere $\alpha^{\prime \prime}$ mit $\alpha$ oder $\beta$; ersteres, dass nämlich $\alpha^{\prime \prime}$ mit $\alpha$ coincidirt, kann wiederum nicht der Fall sein, denn sonst hätten wir ein Arrangement, wie folgt

$$
\begin{aligned}
& \alpha \quad \text { träfe } a b c d e f \\
& \beta \quad-\quad a b c d \\
& \gamma \quad-\quad a b c e f
\end{aligned}
$$

was nicht möglich ist aus dem vorhin angegebenen Grunde; es muss also $\alpha^{\prime}$ mit $\alpha, \beta^{\prime}$ mit $\gamma, \alpha^{\prime \prime}$ mit $\beta$ und $\beta^{\prime \prime}$ mit $\gamma$ zusammenfallen, d. $h$.

$$
\begin{aligned}
& \alpha \text { trifft } a b c d e \\
& \beta-a b c d f \\
& \gamma \quad-\quad a b c e f, \quad \text { also: }
\end{aligned}
$$

Die sechs Geraden $a b$ b $d$ e fliegen nothwendig so, dass, wenn irgend vier von ihnen herausgenommen werden, die eine derjenigen beiden Geraden, welche diesen vieren gleichzeitig begegnen, zugleich einer der beiden übrig gebliebenen und die andere der anderen begegnen muss oder so, dass irgend füf von den Geraden $a b$ b $d$ e $f$ von einer und nur einer einzigen getroffen werden.

5. Da sich die sechs anfänglichen Geraden nur auf sechs Arten zu je fünf combiniren lassen, so erhalten wir also nur sechs neue Gerade, von denen drei die obigen $\alpha \beta \gamma$ waren. Wir bezeichnen jetzt diese sechs neuen Geraden der Fläche $F^{(3)}$ durch $a_{1} b_{1} c_{1} d_{1} e_{1} f_{1}$ und zwar so, dass

$$
\begin{aligned}
& a_{1} \text { trifft } b \quad c \quad d e f \\
& b_{1}-a c d e f \\
& c_{1}-a b d e f \\
& d_{1}-a b c e f \\
& e_{1}-a b c d f \\
& f_{1}-a b c d e \text {, }
\end{aligned}
$$


woraus denn zugleich ein reciprokes Verhalten hervorgeht, dass nämlich

$$
\begin{array}{lcccccc}
a & \text { trifft } & b_{1} & c_{1} & d_{1} & e_{1} & f_{1} \\
b & - & a_{1} & c_{1} & d_{1} & e_{1} & f_{1} \\
c & - & a_{1} & b_{1} & d_{1} & e_{1} & f_{1} \\
d & - & a_{1} & b_{1} & c_{1} & e_{1} & f_{1} \\
e & - & a_{1} & b_{1} & c_{1} & d_{1} & f_{1} \\
f & - & a_{1} & b_{1} & c_{1} & d_{1} & e_{1}
\end{array}
$$

Aus diesen 12 Geraden ergeben sich nun sofort die funfzehn übrigen, denn die beiden Ebenen

$$
\left(\begin{array}{lll}
a & \left.b_{1}\right) \text { und }\left(b a_{1}\right)
\end{array}\right.
$$

schneiden sich in einer Geraden $g_{12}$, welche ganz der Fläche $F^{(3)}$ angehören muss, weil sie gleichzeitig den vier Geraden $\begin{array}{llllll}a & b & a_{1} & b_{1} & \text { begegnet; es kommen }\end{array}$ also zu den früheren 12 noch $\frac{6.5}{1.2}=15$ neue Gerade $g_{i k}$; zusammen giebt es also 27 Gerade auf der Oberfläche dritter Ordnung.

Die letzteren funfzehn Geraden liegen mit den ersteren zwölf in $2.15=30$ solchen Ebenen $A$, deren jede drei der Oberfläche angehörige Gerade enthält, nämlich in einer Ebene liegen immer

$$
\text { (I.) } \quad\left(\begin{array}{lll|lll}
a & b_{1} & g_{12} & a_{1} & b & g_{12} \\
a & c_{1} & g_{13} & a_{1} & c & g_{13} \\
a & d_{1} & g_{14} & a_{1} & d & g_{14} \\
a & e_{1} & g_{15} & a_{1} & e & g_{15} \\
a & f_{1} & g_{16} & a_{1} & f & g_{16} \\
b & c_{1} & g_{23} & b_{1} & c & g_{23} \\
b & d_{1} & g_{24} & b_{1} & d & g_{24} \\
b & e_{1} & g_{25} & b_{1} & e & g_{25} \\
b & f_{1} & g_{26} & b_{1} & f & g_{26} \\
c & d_{1} & g_{34} & c_{1} & d & g_{34} \\
c & e_{1} & g_{35} & c_{1} & e & g_{35} \\
c & f_{1} & g_{36} & c_{1} & f & g_{36} \\
d & e_{1} & g_{45} & d_{1} & e & g_{45} \\
d & f_{1} & g_{46} & d_{1} & f & g_{46} \\
e & f_{1} & g_{56} & e_{1} & f & g_{56}
\end{array} .\right.
$$

Dass aus der Gruppe der 12 Geraden $a b \ldots f, a_{1} b_{1} \ldots f_{1}$ sich keine neuen Geraden herleiten lassen, welche ganz der Oberfläche $F^{(3)}$ angehören könnten, ist ersichtlich; die funfzehn Geraden $g_{i k}$ sind aber selbst Bedingungen unter- 
worfen, aus denen hervorgeht, dass auch sie auf keine weiteren Geraden führen können, so dass dann erwiesen ist, dass die Fläche $F^{(3)}$ im Allgemeinen nur jene 27 Geraden haben kann.

Nehmen wir nämlich zwei Ebenen, z. B.

$$
\begin{array}{lllllll}
a & b_{1} & g_{12} & \text { und } & c & d_{1} & g_{34} \text {, }
\end{array}
$$

aus dem obigen Schema (I) heraus, so wird ihre Schnittlinie der Fläche $F^{(3)}$ im Allgemeinen in drei Punkten begegnen, von denen zwei offenbar der Schnittpunkt von $a d_{1}$ und der Schnittpunkt von $b_{1} c$ sind; der dritte Schnittpunkt muss daher sowohl in $g_{12}$ als auch in $g_{34}$ liegen, $d$. h. die beiden Geraden $g_{12}$ und $g_{34}$ müssen sich schneiden und im Allgemeinen müssen immer $g_{i k}$ und $g_{i^{\prime} k^{\prime}}$ in einer Ebene liegen, sobald $i k, i^{\prime} k^{\prime}$ von einander verschieden sind. Dass die Schnittlinie der betrachteten beiden Ebenen nicht ganz auf der Oberfläche $F^{(3)}$ liegen kann, ist kaum zu erwähnen nöthig, weil sonst eine Ebene mit der Fläche $F^{(3)}$ vier gerade Linien gemein hätte, was unmöglich ist.

Da $g_{12}$ und $g_{34}$ in einer Ebene liegen, so muss diese der Fläche $F^{(3)}$ nothwendig noch in einer dritten geraden Linie begegnen, die leicht anzugeben ist, nämlich $g_{56}$; denn letztere schneidet nach dem Vorigen sowohl $g_{12}$ wie $g_{34}$, liegt folglich mit diesen beiden sich selbst schneidenden Geraden in einer Ebene, und im Allgemeinen liegen immer drei Gerade $g_{i_{k}} g_{i^{\prime} k^{\prime}} g_{i^{\prime \prime} k^{\prime \prime}}$ in derselben Ebene, bei welchen $i k, i^{\prime} k^{\prime}, i^{\prime \prime} k^{\prime \prime}$ von einander verschieden sind. Hieraus ergeben sich 15 neue Ebenen $\Delta$, in welche sich die Geraden $g_{i k}$ folgendermaassen vertheilen:

(II.) $\quad \begin{array}{lll}g_{12} & g_{34} & g_{56} \\ g_{12} & g_{35} & g_{46} \\ g_{12} & g_{36} & g_{45} \\ g_{13} & g_{24} & g_{56} \\ g_{13} & g_{25} & g_{46} \\ g_{13} & g_{26} & g_{45} \\ g_{14} & g_{23} & g_{56} \\ g_{14} & g_{25} & g_{36} \\ g_{14} & g_{26} & g_{35} \\ g_{15} & g_{23} & g_{46} \\ g_{15} & g_{24} & g_{36} \\ g_{15} & g_{26} & g_{34} \\ g_{16} & g_{23} & g_{45} \\ g_{16} & g_{24} & g_{35} \\ g_{16} & g_{25} & g_{34} .\end{array}$


Es giebt also, wenn wir die früheren 30 Ebenen hinzurechnen, im Ganzen $30+15=45$ solche Ebenen 4 , deren jede drei von den 27 Geraden enthält. Aus diesen Beziehungen der Geraden $g_{i k}$ unter einander oder mit den anfänglichen 12 Geraden geht hervor, dass sich keine anderen Geraden weiter auf der Fläche $\boldsymbol{F}^{(3)}$ ziehen lassen.

6. Wählen wir von den eben erhaltenen 45 Ebenen $\Delta$ zwei aus, welche sich nicht in einer der 27 Geraden schneiden, z. B.

$$
\begin{array}{lll}
g_{12} & g_{34} & g_{56} \\
g_{46} & g_{25} & g_{13},
\end{array}
$$

so liegen die so unter einander gestellten Geraden selbst paarweise in einer Ebene, welche der Fläche $F^{(3)}$ in je einer dritten Geraden begegnet; diese hinzugefügt erhalten wir folgendes Schema

\begin{tabular}{c|c|c}
$g_{12}$ & $g_{34}$ & $g_{56}$ \\
\hline$g_{46}$ & $g_{25}$ & $g_{13}$ \\
\hline$g_{35}$ & $g_{16}$ & $g_{24}$,
\end{tabular}

bei welchem immer drei in einer Horizontal - und gleichzeitig drei in einer Vertical-Reihe stehende Gerade in je einer Ebene liegen; die drei ersten Ebenen sind die Flächen eines Steinerschen Trieders und die drei anderen des conjugirten Trieders; die 9 Geraden, in welchen die Flächen der beiden conjugirten Trieder einander schneiden, gehören sämmtlich der Fläche $F^{(3)}$ an. Man kann ein solches Triederpaar auch aus dem Schema (I.) bilden und zwar in zweifacher Weise, wofür folgende Beispiele Prototypen sind:

1)

$\left\{\begin{array}{c|c|c}a & b_{1} & g_{12} \\ \hline c_{1} & d & g_{34} \\ \hline g_{13} & g_{24} & g_{56}\end{array}\right.$

2)

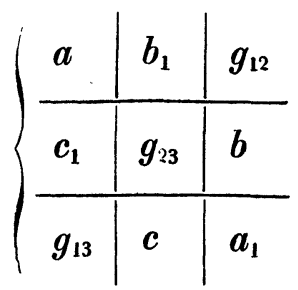

Fragen wir, wie viel Triederpaare sich überhaupt bilden lassen, so ergiebt sich zunächst aus dem Schema (I.) der 30 Ebenen $\Delta$ nach dem Modell 1), dass wir so viel demselhen analoge bilden können, als sich sechs Elemente zu je vier combiniren lassen, also $\frac{6.5 .4 .3}{1 \cdot 2.3 .4}=15$, und aus jeder solchen Ver- 
bindung von je vier Elementen immer 12 Triederpaare hervorgehen, z. B. $\begin{array}{llllllllll}\text { aus } a & b & c & d & a b_{1} & a b_{1} & a c_{1} & a c_{1} & a d_{1} & a d_{1}\end{array}$ $\begin{array}{llllll}c_{1} d & d_{1} c & b_{1} d & d_{1} b & b_{1} c & c_{1} b\end{array}$ $\begin{array}{llllll}a_{1} b & a_{1} b & a_{1} c & a_{1} c & a_{1} d & a_{1} d\end{array}$ $\begin{array}{cccccc}c d_{1} & d c_{1} & b d_{1} & d b_{1} & b c_{1} & c b_{1},\end{array}$

wo die zugehörigen $g_{i k}$ der neun, das Triederpaar constituirenden Terme leicht zu ergänzen sind. Von diesen $15.12=180$ Triederpaaren sind aber die Hälfte fortzulassen, weil immer zwei auftreten, die nur dadurch verschieden sind, dass Horizontal - und Verticalreihen mit einander vertauscht sind, wobei natürlich das Triederpaar dasselbe ist, z. B.

$$
\begin{array}{lllll}
a & b_{1} & \text { und } & a & c_{1} \\
c_{1} & d
\end{array} \quad \begin{array}{lll}
b_{1} & d
\end{array}
$$

Zu diesen 90 Triederpaaren kommen nun diejenigen, welche aus dem Modell 2) hervorgehen, und deren es offenbar so viel giebt, als sich sechs Elemente zu je drei combiniren lassen, d. h. $\frac{6.5 .4}{1.2 .3}=20$; endlich noch diejenigen, welche sich aus der Gruppe (II.) der 15 Ebenen $\Delta$ bilden lassen. Hier ist jede Ebene nur mit 8 anderen so zu verbinden, dass sich diese beiden nicht in einer der 27 Geraden schneiden, und dies giebt $\frac{15.8}{2}=60$ Combinationen, welche auf Triederpaare führen; hiervon ist aber nur der sechste Theil zu nehmen, weil sich sowohl Horizontal- und Vertical-Reihen mit einander vertauschen lassen, als auch jede dieser drei Reihen auf drei Arten zu je zweien mit einander verbunden werden können; wir erhalten mithin nur 10 neue Triederpaare, die aus je neun $g_{i k}$ zu bilden sind und hiernach im Ganzen $90+20+10=120$ Triederpaare. Verschiedene Ebenen aus den Gruppen (I.) und (II.) mit einander in Verbindung gebracht, führen natürlich wieder zu Triedern, die zu den ersten 90 gehören.

7. Aus sämmtlichen 27 Geraden der Fläche $F^{(3)}$ lassen sich noch auf mehrfache Weise solche 6 Paare auswählen, wie diejenigen waren, auf welche wir zuerst geführt wurden, nämlich:

$$
\text { (I.) }\left\{\begin{array}{llllll}
a & b & c & d & e & f \\
a_{1} & b_{1} & c_{1} & d_{1} & e_{1} & f_{1}
\end{array}\right.
$$

wo zwei unter einander stehende sich nicht schneiden, dagegen jede der einen Reihe die fünf anderen mit Ausnahme der mit ihr in derselben Colonne stehenden trifft.

In der That ergiebt sich aus der Anschaung der obigen Beziehungen zwischen den 27 Geraden, dass die 12 Geraden 


$$
\text { (II.) } \quad\left\{\begin{array}{llllll}
a & a_{1} & g_{23} & g_{24} & g_{25} & g_{26} \\
b & b_{1} & g_{13} & g_{14} & g_{15} & g_{16}
\end{array}\right.
$$

eine ganz ähnliche Gruppe von 12 Geraden bilden wie die anfängliche; derartiger Gruppen giebt es offenbar $\frac{6.5}{1.2}=15$; ferner erhalten wir noch ein drittes System von 12 Geraden in folgender Weise:

$$
\text { (III.) } \quad\left\{\begin{array}{llllll}
a & b & c & g_{56} & g_{46} & g_{45} \\
g_{23} & g_{13} & g_{12} & d_{1} & e_{1} & f_{1},
\end{array}\right.
$$

und solcher Gruppen giebt es $\frac{6.5}{1.2 .3}=20$, mithin im Ganzen $1+15+20=36$ Gruppen von solchen 12 Geraden, wie die anfänglichen $a b \ldots f, a_{1} b_{1} \ldots f_{1}$. Dass es aber nicht mehr derartiger Gruppen geben kann, ist leicht erkennbar.

Hieraus geht hervor, wie viel Hyperboloide $\boldsymbol{H}^{(2)}$ es im Allgemeinen giebt, welche der Oberfläche $F^{(3)}$ in je 6 Geraden begegnen; denn nehmen wir bei einer beliebigen der erhaltenen 36 Gruppen aus der ersten Reihe drei Gerade heraus, so muss das durch dieselben gelegte Hyperboloid $\boldsymbol{H}^{(2)}$ die drei anderen Geraden der zweiten Reihe, welche nicht unter ihnen stehen, mit der Fläche $F^{(3)}$ gemein haben, und da sich 6 Elemente zu je dreien auf $\frac{6.5 .4}{1.2 .3}=20$ Arten combiniren lassen, so müsste es $20.36=720$ Hyperboloide $H^{(2)}$ geben, deren jedes der Fläche $F^{(3)}$ in 6 Geraden begegnet; diese treten aber doppelt auf, so dass es nur 360 wirklich von einander verschiedene Hyperholoide $H^{(2)}$ giebt. Hiervon überzeugt man sich leicht aus der Anschauung der 3 Schemata (I.), (II.), (III.), ohne nöthig zu haben, dieselben vollständig auszuführen, denn es lassen sich immer aus den ersten Reihen jener drei Schemata je drei das Hyperboloid $\boldsymbol{H}^{(2)}$ bestimmende Generatrices derselben Art, wie sie auch immer gewählt seien, entweder in demselben Schema noch einmal oder in einem der anderen Schemata wiederfinden, nämlich

$$
\begin{array}{lllll}
a & b & c & \text { in (I.) } & \text { und (III.) } \\
a & a_{1} & g_{23} & \text { in (II.) } & \text { und (II.) } \\
a & g_{23} & g_{24} & \text { in (II.) } & \text { und (III.) } \\
a & b & g_{56} & \text { in (III.) und (III.) } \\
g_{56} & g_{46} & g_{45} & \text { in (III.) und (III.). }
\end{array}
$$

In eine dieser 5 Kategorien ist aber jedes Hyperboloid $\boldsymbol{H}^{(2)}$, wie die Generatrices auch immer ausgewählt seien, welche es bestimmen, unțerzubringen; es kommt daher jedes doppelt vor und die Anzahl der von einander wirklich verschiedenen betrăgt 360 . 
8. Aus den beiden Gruppen (I.) und (II.) in $\mathbf{n}^{\circ} .5$, worin die $27^{\circ}$ Geraden in 30 und 15 Ebenen vertheilt sind, geht hervor, dass jede dieser Geraden von 10 anderen getroffen wird, z. B.

$$
\begin{array}{lllllllllllll}
a & \text { von } & b_{1} & c_{1} & d_{1} & e_{1} & f_{1} & g_{12} & g_{13} & g_{14} & g_{15} & g_{16}
\end{array}
$$

und

$$
\begin{array}{llllllllllll}
g_{12} & \text { von } & a & a_{1} & b & b_{1} & g_{34} & g_{35} & g_{36} & g_{45} & g_{46} & g_{56},
\end{array}
$$

aber von weiter keiner; diese 10 Schnittpunkte rühren von 5 Paaren Gerader her, deren jedes in einer Ebene enthalten ist und die als 5 Paare aufgefassten Schnittpunkte bilden ein Punktsystem (Involution) wie aus folgender Betrachtung hervorgeht.

Ein durch $e \quad f g_{12}$ gelegtes Hyperboloid $H^{(2)}$ begegnet der Fläche $F^{(3)}$ in

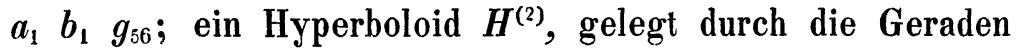

e $f g_{13}$, begegnet der Fläche $F^{(3)}$ in den Geraden

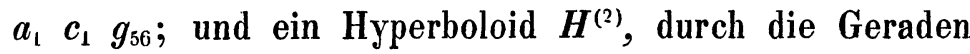

$e \quad f \quad g_{14}$ gelegt, begegnet der Fläche $F^{(3)}$ in $\begin{array}{llll}a_{1} & d_{1} & g_{56}\end{array}$

Diese drei Hyperboloide haben vier Generatrices gemein $e f a_{1} g_{56}$ und schneiden die Fläche $F^{(3)}$ ausserdem in den Linienpaaren $b_{1} g_{12}, c_{1} g_{13}, d_{1} g_{14}$, welche selbst der Geraden $a$ in drei Punktenpaaren begegnen. Ausserdem gehen durch die vier Geraden $e f a_{1} g_{56}$ zwei Ebenenpaare, nämlich einmal $e a_{1}$ und $f g_{56}$ und zweitens $e g_{56}$ und $f a_{1}$, und das erste Ebenenpaar begegnet der Geraden $a$ in denjenigen beiden Punkten, in welchen sie von $e_{1}$ und $g_{15}$ getroffen wird, das andere Ebenenpaar aber in denjenigen beiden Punkten, in welchen sie von $f_{1}$ und $g_{16}$ getroffen wird; betrachten wir die beiden Ebenenpaare als besondere Flächen zweiten Grades, so haben wir durch die vier Geraden e f $a_{1} g_{56}$ (besonderer Fall einer Raumcurve vierten Grades) fünf Flächen zweiten Grades, welche bekanntlich einer Geraden $(a)$ in fünf Punktenpaaren eines Punktsystems begegnen; folglich bilden die fünf Paar Schnittpunkte, in welchen eine jede der 27 Geraden von füf Paaren der übrigen, die immer paarweise in einer Ebene liegen, getroffen werden, ein Punktsystem. In ganz ähnlicher Weise, wie hier der Beweis für die Gerade $a$ geführt ist, lässt er sich auch für eine der Geraden $g_{i k}$ führen, worauf einzugehen unnöthig ist. Durch die vier Geraden ef $a_{1} g_{56}$, welche ein schiefes Viereck bilden, gehen ausser den beiden Ebenenpaaren und den drei angeführten Hyperboloiden $\boldsymbol{H}^{(2)}$ noch eine unendliche Zahl anderer Hyperboloide $h^{(2)}$, die ein einfaches Flächenbüschel bilden. Jedes Hyperboloid dieses Büschels begegnet 
der Geraden $a$ in zwei Punkten $p, \pi$ eines Punktsystems, welches schon durch die beiden Ebenenpaare fixirt wird; ein solches Hyperboloid $h^{(2)}$ kann aber mit der Fläche $F^{(3)}$ im Allgemeinen nur noch einen Kegelschnitt $C^{(2)}$ gemein haben, welcher daher durch die Punkte $p, \pi$ hindurchgehen, folglich mit der Geraden $a$ in derselben Ebene liegen muss. Die Ebenen aller Kegelschnitte $C^{(2)}$ bilden also ein Ebenenbüschel $B(a)$. Wir schliessen hieraus umgekehrt: Alle Ebenen durch eine der 27 Geraden gelegt, schneiden die Fläche $F^{(3)}$ längs Kegelschnitten $C^{(2)}$, welche selbst wieder der ersten Geraden in Punktenpaaren eines Punktsystems begegnen. Insbesondere zerfällt der Kegelschnitt $C^{(2)}$, wie wir gesehen haben, fünfmal in Linienpaare, die 5 Punktenpaare jenes Punktsystems auf der Geraden fixiren. Das Ebenenbüschel $\boldsymbol{B}(\boldsymbol{a})$ steht mit dem Flächenbüschel $B\left(h^{(2)}\right)$ in projectivischer Beziehung, denn es gehört sowohl zu jedem Hyperboloid $h^{(2)}$ eine einzige und bestimmte Ebene des Ebenenbüschels als auch umgekehrt zu jeder Ebene des Ebenenbüschels ein einziges und bestimmtes Hyperboloid $h^{(2)}$; letzteres folgt nämlich daraus, dass eine durch $a$ gelegte Ebene der Fläche $F^{(3)}$ noch in einem bestimmten Kegelschnitte $C^{(2)}$ begegnet, welcher die Gerade $a$ selbst in einem bestimmten Punktenpaare $p, \pi$ des obigen Punktsystems trifft; durch dies Punktenpaar und die vier Geraden e $f a_{1} g_{56}$ ist aber nur ein einziges Hyperboloid $h^{(2)}$ möglich, welches eben das der Ebene entsprechende ist; da diese Beziehung eine beiderseitig eindeutige ist, so muss sie bekanntlich projectivisch sein.

Hieraus geht ein besonderer Fall der von Steiner (a. a. 0. II) angegebenen Entstehungsart der Oberfläche dritter Ordnung hervor mittelst eines Ebenenbüschels und eines mit ihm projectivischen Büschels von Flächen zweiter Ordnung, welche durch dieselbe Raumcurve $\boldsymbol{R}^{(4)}$ hindurchgehen, indem nämlich an Stelle dieser Raumcurve $\boldsymbol{R}^{(4)}$ die Seiten eines schiefen Vierecks treten und das Flächenbüschel aus sämmtlichen durch dasselbe gehenden Hyperboloiden besteht. Dieser Besonderheit wegen wird die Construction der Oberflächè dritter Ordnung von elementarer Einfachheit und lässt sich so aussprechen: Durch zwei einander nicht schneidende Gerade $a$ b und zwei andere einander ebenfalls nicht schneidende Gerade $\alpha \beta$, die aber jede der beiden ersten treffen, durch eine fünfte Gerade c, welche keiner der vier begegnet, und drei beliebige Punkte im Raume ist eine Oberfläche dritter Ordnung vollständig bestimmt und kann folgendermaassen construirt werden: Man lege durch $a \quad b \quad \alpha \quad \beta$ und je einen der drei gegebenen Punkte drei Hyperboloide und lasse sie den drei Ebenen, welche durch $c$ und dieselben drei Punkte 
gehen, projectivisch entsprechen; durch diese drei entsprechenden Paare ist die projectivische Beziehung des Ebenenbüschels und Hyperboloidenbüschels vollständig bestimmt; jede vierte durch $c$ gelegte Ebene wird das bestimmte ihr entsprechende Hyperboloid längs eines Kegelschnittes schneiden, dessen gesammter Ort, die Oberfläche dritter Ordnung ist. Oder auch: Durch das Hyperboloidenbüschel $(a b \alpha \beta)$ ist auf der Geraden $c$ ein bestimmtes Punktsystem $p, \pi$ gegeben; man lege durch $c$ ein Ebenenbüschel, welches mit dem Punktsystem $p, \pi$ in projectivische Beziehung gesetzt wird durch drei Paar als entsprechend festgesetzte Elemente; das durch ein Punktenpaar $p, \pi$ gelegte Hyperboloid des Flächenbüschels $(a b \alpha \beta)$ und die entsprechende Ebene des Ebenenbüschels schneiden sich längs einem Kegelschnitte, dessen gesammter Ort die Oberfläche dritter Ordnung ist.

Diese Construction kommt gleichzeitig mit der von F. August a. a. 0. gegebenen Definition der Oberfläche dritter Ordnung überein; denn jedes Hyperboloid unseres Flächenbüschels lässt sich als das Erzeugniss zweier projectivischen Ebenenbüschel auffassen, deren Axen $a$ und $b$ sind; einer bestimmten durch $c$ gehenden Ebene entsprechen also zwei bestimmte projectivische Ebenenbüschel, deren Axen $a$ und $b$ sind (a. a. 0. \$ 4) und bewegen wir die Ebene um die dritte Axe $c$ herum, so lassen sich $a b c$ als die Axen dreier sogenannter „doppelt-projectivischer" Ebenenbüschel auffassen, wie sie August seiner Betrachtung zu Grunde gelegt hat.

Die Oberfläche dritter Ordnung als Erzeugniss dreier collinearen Ebenenbündel aufgefasst kann unmittelbar durch drei beliebige Punkte (als Mittelpunkte der drei Bündel) und vier beliebige Gerade im Raume, von denen sich nicht zwei begegnen und die auch nicht die vier Generatrices derselben Art eines Hyperboloids sind, gelegt werden, weil die collineare Beziehung zweier Bündel durch vier Paar entsprechende Elemente bestimmt ist. Aus derselben Entstehungsart der Oberfläche dritter Ordnung geht auch ein von Salmon aufgestellter Satz unmittelbar hervor (Nouvelles annales de mathématiques par M. M. Gerono et Prouhet II ${ }^{\text {me }}$ série, tome II, pag. 24), der einem bekannten Satze der Ebene analog ist:

Wenn die vier Flächen eines Tetraeders durch feste Punkte laufen und die drei Kanten einer Seitenfläche auf festen Ebenen sich bewegen, so beschreibt die dieser Seitenfläche gegenüberliegende Ecke des Tetraeders eine Oberfläche dritten Grades.

Breslau, den $28^{\text {sten }}$ März 1863. 